that the measurements given below were obtained by projecting, by aid of a $\mathbf{1}^{\prime \prime}$ objective, the image of a diamond, which was rather less than $2 \mathrm{~mm}$, in extreme length, into a camera, so that it was enlarged upon the screen to II c.m. across. Two micrometer eye-pieces with movable wires were directed upon opposite corners of the image, the diamond being manipulated in the field of the object-glass till these corners were in sbarp focus. A movement of the wires of the reading microscopes by one division of the head of the micrometer in following the expansion of the image corresponded to a diametral enlargement of this latter by $0.0005 \mathrm{c.m}$., but owing to. imperfect focus of the image no more than o.oor could be accurately determined. If the coefficient of expansion of the substance were 0.00001 (that of platinum, about) the expansion of the image, due to $10^{\circ} \mathrm{C}$. change of temperature, is just determinable. It will therefore be understood that over a wide range of tem. perature considerable certainty in the readings could be secured.

The heating w'as effected by radiation from a platinum ribbon folded in such a manner that the substance under observation occupied the central point of a narrow platinum tube. The ribbon was heated by a current. A beam of light from a very small sphere $-2 \mathbf{~ m m}$. in diameter - of incandescent lime (heated in an oxyhydrogen flame) entered the tubular oven from the back. The adjustment of this beam greatly decided the sharpness of the projected image. Temperatures were determined

served by crystalline forces which will require to be brought into play by external conditions of pressure. It is probable that this is therefore an essential condition of success in its artificial production. It is perhaps of little interest to add that this reasoning gave rise to experiments-as I had leisure for themwhich I only laid aside finally upon hearing of M. Moissan's success. I did not seek the aid of solution in a metal, but used an apparatus to compress graphite, as well as carbon prepared from sugar, between iron plates kept at a red heat, and urged together by the alternate heating and cooling of the bars of an iron yoke.

I am not without hope that the use of high pressure at a high temperature may ultimately prove sufficient-without resort to solution in a metal-to produce diamond. If the presence of a certain minute quantity of the carbide of a metal is essential, of course it will fail. It is difficult to imagine, however, that it should be essential.

'Trinity College, Dublin, March 5. J. Joly.

\title{
The North-East Wind.
}

Prof. Bonney, in his "Story of our Planet," explains the prevalence of east winds at this season as being due to the low winter temperature of eastern Europe compared with the Atlantic coasts. If this explanation be the true one, we should expect the phenomenon to occur in December and January. My impression is, although I have no accurate statistics, that east winds do not prevail in those months in our climate. Further, we should expect, crossing the Atlantic in winter, to find for the same reason west winds prevailing off the American coasts. If this is not found to be the case, I would suggest the following as, if not the cause, at all events one of the causes, of the phenomenon in question.

The difference of temperature be. tween the northern Arctic regions and the tropics, to which, combined with the earth's rotation, the north east trade wind is supposed to be due, is necessarily greater in the spring months, February to June, than on the average of the year. Because in the Arctic regions, little or no heat being received directly from the sun between the autumnal and the vernal equinox, the maximum of cold should be attained in March or from February to Aprit. In low latitudes these months are by no means the coldest. It is reason-

FIG. I.

by meiting substances of known melting-points in the oven, and reading the corresponding currents. Thus a curve of galvanometer readings plotted against temperature was obtained for subsequent use.

The results of the observations were four well-fixed points which give a curve (Fig. I) seemingly tangential to Fizeau's results at low temperatures, but which curls up rapidly at about $70^{\circ} \mathrm{C}$. At a temperature of $850^{\circ}$, and indeed below this, observations were stopped by the "efflorescence" upon the surface of the diamond of flaky particles which wriggled and twisted in a peculiar manner, finally disappearing. Once started, the "combustion" continued till the temperature of the oven was lowered to $712^{\circ}$. Cooling the oven, I subsequently photographed one face of the diamond. The picture obtained shows the face with a lamellar appearance, which was produced entirely by the heating, as at starting the faces were smoothly curved. Such an appearance is occasionally observed upon specimens of diamond. This photograph, as well as the curve of expansion, were shown at the soiree of the Royal Society in June 1892 . The apparatus used was also shown in operation as applied to a minute globule of a melted basalt.

The sudden increase in volume or swelling-up of the diamond at high temperatures, suggests that the diamond is a form of carbon which has been subjected to high pressure when crystallising. Such changes we may expect to be reversible, and it is supposable that equilibrium at the higher density is only preNO. I 273 , VOL. 49$]$ able to cxpect that when this difference of temperature becomes accentuated, and the gradients, so to speak, steeper, the north-east winds which are due to it should become prevalent in higher latitudes than those to which the trade winds proper are usually confined.

S. H. BURBURY.

\section{The Suspension of Foreign Bodies from Spiders Webs.}

THE following instance of the use of a stone by a spider as ballast for its web is interesting.

A web was noticed stretched between two trees at a distance of about ten feet from one another. From it hung a thread about two feet long, and attached to its lower end was a small pebble about the size of a pea, the stone hanging free about four feet from the ground. The stone had evidently been made use of in this special manner by the spider for the definite purpose either of keeping the web taut, or as ballast to give it stability against the wind, for on lifting the stone to remove the pressure, it was observed that the web became limp and slack, and was stirred out of position by the least breath of air.

This was noticed by a score or so of members of the German "Turnverein" here, in the garden of whose premises the occurrence took place.

Buenos Ayres, January 24.
R. PHIIIPE. 